\title{
From Supramolecular Photochemistry to Self-Assembled Photoactive Architectures: The Emergence of Photochemical Nanosciences
}

\author{
Dario M. Bassani* \\ Grammaticakis-Neuman Prize Winner 2005
}

\begin{abstract}
The use of supramolecular interactions to control and direct excited-state reactivity is described, with emphasis on the author's contributions in this area. The systems are based on the multiple-point hydrogen-bonding molecular recognition motif present in the melamine-barbituric acid system, and are designed to operate in solution phase. Despite the weak nature of non-covalent interactions, control of high energy intermediates such as excited cinnamantes, stilbenes, or fullerenes is possible. These results demonstrate the particular sensitivity of short-lived electronically excited molecules to their environment, and particularly to the mutual orientation of the reactants.
\end{abstract}

Keywords: Molecular recognition $\cdot$ Photochemistry $\cdot$ Photovoltaic $\cdot$ Self-assembly

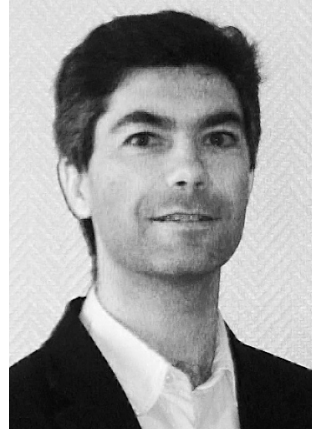

Dario Bassani was born in Milan, Italy, and after obtaining a Licence en Chimie degree from the Université Catholique de Louvain (Belgium), completed a doctoral degree in the field of organic photochemistry in 1993 under the guidance of Professor F.D. Lewis at Northwestern University (USA). He

${ }^{*}$ Correspondence: Dr. D.M. Bassani

Centre de Recherche en Chimie Moléculaire

LCOO CNRS UMR 5802

Université Bordeaux 1

351, Cours de la Libération

33405 Talence France

Tel.: +33 540002827

Fax: +33 540006158

E-Mail: d.bassani@lcoo.u-bordeaux1.fr then undertook a postdoctoral position as a Hoffmann-La Roche fellow with Professor J. Wirz (Basel University, Switzerland), followed by a second postdoctoral position as a Marie Curie Fellow with Professor J.M. Lehn in Strasbourg (France). He joined the Centre National de la Recherche Scientifique in 1997 as research scientist appointed to the Laboratoire de Chimie Organique et Organométallique (CNRS UMR 5802) at Bordeaux University, where he conducts research bridging photochemistry and supramolecular chemistry. $\mathrm{He}$ is the recipient of the Inter-American Photochemical Society Young Investigator Award (2003), the French Chemical Society Award (Physical Chemistry Division, 2004), and the Grammaticakis-Neuman Prize of the Swiss Chemical Society (2005).

\section{Introduction}

In recent years, the development of nanoscale molecular devices has emerged as a viable route to intelligent functional materials operating at the molecular level. Light plays an important role in many of the exploratory devices that are being conceived, being capable of supplying both energy for operation and input/output signals to the macroscopic world. Because light-induced processes are extremely fast, they are very sensitive to the three-dimensional orienta- tion of the components. This well-known observation dates back to the early days of photochemistry and the exploration of the solid-state reactivity of cinnamic acid, to be later formulated by Schmidt and co-workers into the principle of topological control [1]. Examples of solid-state photochemical transformations, particularly photocycloadditions, known to be under topochemical control are much more abundant than their counterparts in solution [2]. This is in part due to the availability of X-ray diffraction data yielding precise molecular distances and orientations of the reactants and, in some cases, it has been possible to favor the formation of certain products by the inclusion of molecules capable of inducing specific geometrical constraints [3]. In contrast, efforts to precisely design molecular components so that they will spontaneously organize into a photoreactive assembly in solution are frustrated by such factors as background reactivity and the difficulty in demonstrating the intervention of the proposed architecture.

We thus turned our attention to investigate how the use of directional non-covalent interactions, particularly hydrogenbonding, could be used to direct and control light-induced processes. Our initial work dealt with $[2+2]$ photodimerization reactions as they present the added interest of producing rigid cyclobutane structures that retain the relative geometry of the reactants, 
thus 'capturing' the supramolecular assembly [4]. The study of organizational effects on other processes, such as energy- and electron transfer, is of interest for the design and fabrication of molecular electronic devices for light-to-electrical energy conversion and o-LED applications. Finally, the use of isomerizable groups to influence the aggregation properties of photosensitive amphiphiles leads to light-to-mechanical force transduction and photoresponsive vesicles for drug delivery [5].

\section{Supramolecular Control of Cinnamate Photodimerization}

By combining a molecular recognition unit with a photodimerizable chromophore, it is possible to use a template molecule possessing complementary recognition motifs to bind and orient the substrates during cycloaddition. This has been demonstrated for a cinnamate ester or stilbene chromophore appended to a triaminotriazine unit [4][6]. The template, a barbituric acid derivative, serves to direct the preferential formation of head-to-head photodimers with a switch in regioselectivity compared to solution (Scheme 1).

In principle the $[2+2]$ cyclodimerization of cinnamates can yield eleven different products, which are commonly divided into two classes, i.e. those arising from a head-to-head dimerization (truxinates) and those from a head-to-tail dimerization (truxillates). Whereas the preparation of $\alpha$ and $\beta$-forms from the irradiation of solid samples is straightforward, the remaining cyclobutane structures are typically more challenging to prepare.

Irradiation of degassed solutions (350 $\mathrm{nm}$, in $\mathrm{CHCl}_{2}, 10^{-2} \mathrm{M}$ ) of $\mathbf{1}$ was carried out in the absence of template, where E/Zisomerization rapidly leads to a photostationary state (two-thirds $Z$-cinnamate isomer). HPLC analysis of the photoproducts after prolonged irradiation indicates that one major and six minor products form in addition to the $Z$-cinnamante isomer. Under identical experimental condition, but in the presence of template 2 ( 0.5 equiv.), the rates of formation for three of the photoproducts are greatly enhanced. These products were isolated and identified as the neotruxinate, $\beta$-truxinate, and $\varepsilon$-truxillate cycloadducts, with the major product being the syn headto-head ( $\beta$-truxinate) dimer. The influence of the template is sufficiently strong to induce the formation of otherwise disfavored products (including the rare head-to-tail $\varepsilon$-truxillate dimer), supporting the conclusion that the photodimerization is under topochemical control within the supramolecular assembly. Overall yields of the three predominant photodimers in the presence of 2 correspond to 3 to 10 -fold enhance-

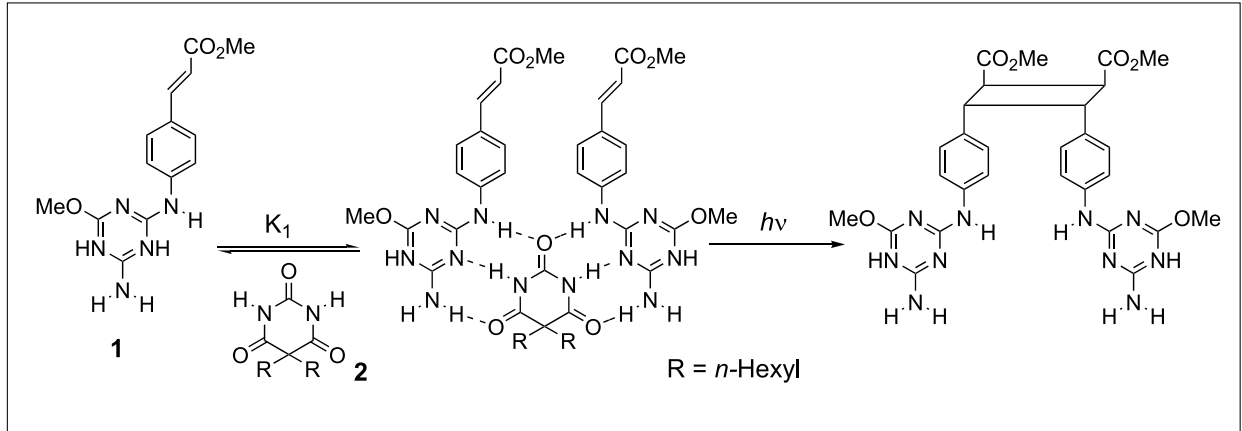

Scheme 1. A barbituric acid derivative serves as a supramolecular template to bind two cinnamate chromophores in a geometry suitable to undergo photoinduced [2+2] dimerization [4] ments, with the quantum yield for the capture of the $\beta$-truxinate within the assembly estimated to be $c a$. 0.06 , at least a 75 -fold increase compared to solution [4b].

The occurrence of selectivity in addition to rate enhancement is an indication that the observed catalytic effect is not due simply due to an increased local concentration of reactants through a form of non-specific aggregation promoted by the barbiturate unit. The preference for photodimers in which the triazine units are syn-oriented is in agreement with the involvement of a supramolecular structure in which 2 acts as a template during the photodimerization process, as illustrated in Fig. 1.

\section{Synergy in the Excited State}

In contrast to the many examples of multiple-input photoactive sensors and switches known to date, examples of photochemical reactions sensitive to multiple chemical inputs are scarce. Compound $\mathbf{3}$ (Scheme 2) was prepared using Heck coupling methodology, and is designed to incorporate a hydrogen-bonding site and a metal ion-binding unit around a photoactive cinnamate chromophore [7].

The tetraethyleneglycol unit in $\mathbf{3}$ is apt to bind alkali metal ions in a fashion similar to well-known podand systems. In the case of metal ions capable of binding

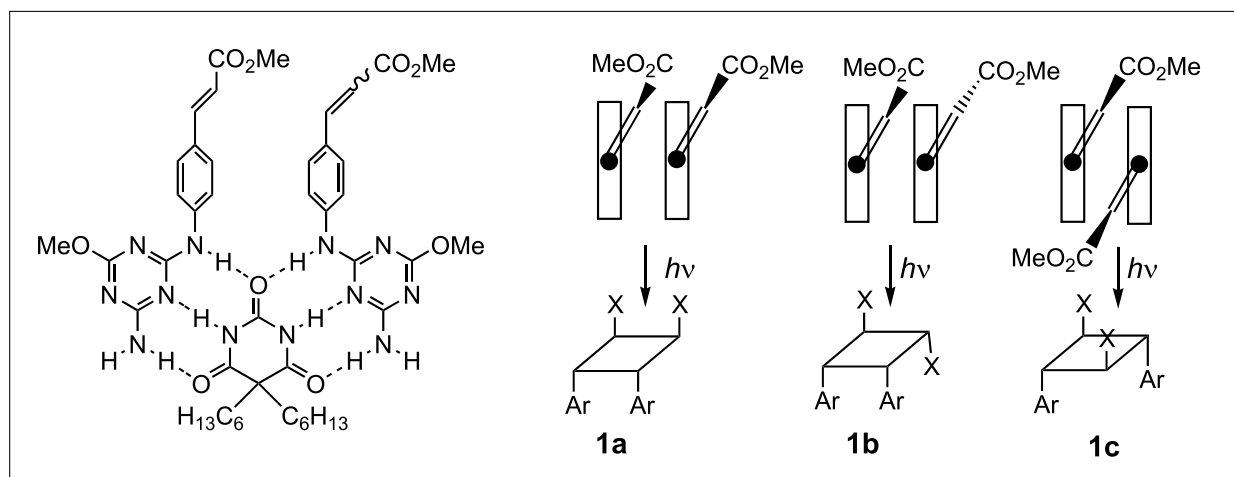

Fig. 1. The relative orientation (syn vs. anti) of the exocyclic $\mathrm{C}=\mathrm{C}$ bond of $\mathbf{1}$ in the trimeric hydrogenbonded assembly on the left determines the formation of photoproducts 1a-1c [4]

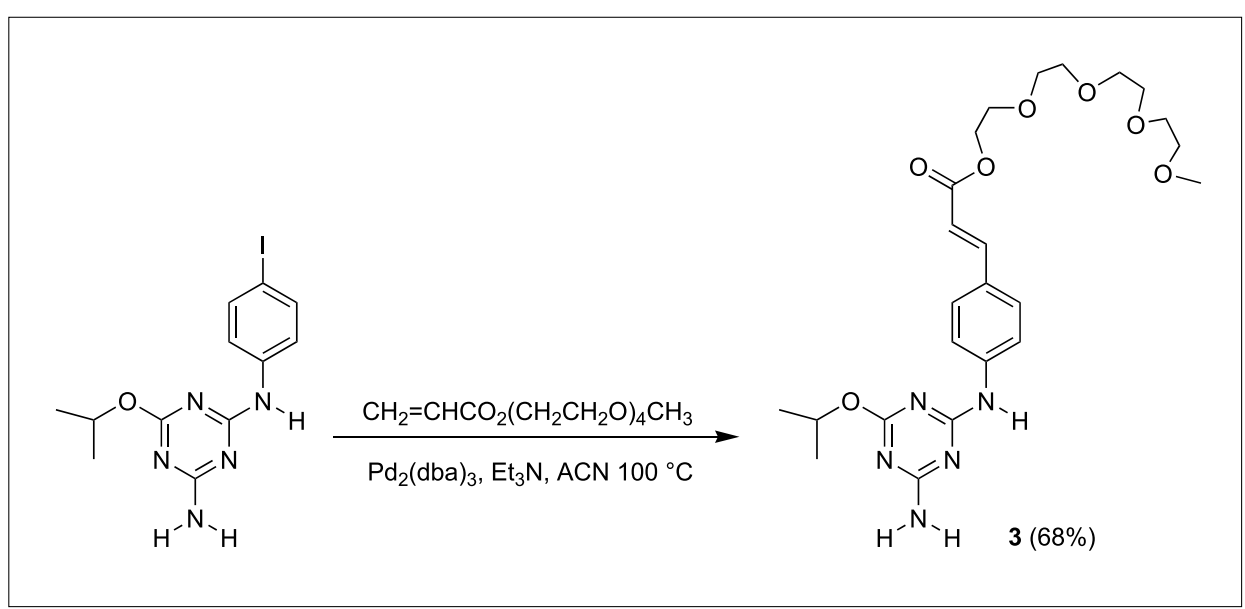

Scheme 2. Synthesis of a photoactive cinnamate receptor possessing binding sites for metal ions and barbiturates such as 2 [7] 
two or more molecules of $\mathbf{3}$, an enhancement in the rate of photodimerization can be expected. This is indeed the case as the formation of photoproducts upon irradiation of 3 in the presence of 3 equiv. $\mathrm{KPF}_{6}$ proceeds $c a$. twice as fast as in the absence of added salt. Interestingly, the combination of 2 and $\mathrm{K}^{+}$gives a five-fold enhancement in dimerisation efficiency, consistent with an additive effect from both molecular receptor units in 3 working in unison. Fig. 2 lists the effect of some group I and group II metal ions upon the dimerisation efficiency of 3 in the presence of 0.5 equiv. of 2 . It is immediately apparent that the system is selective in response to the presence of certain metal ions, particularly $\mathrm{Ba}^{2+}$. Compared to $\mathrm{K}^{+}$, the smaller $\mathrm{Na}^{+}$ion gives a modest enhancement in photoreactivity. No discernable effect upon addition of quaternary ammonium salts could be detected, thus ruling out a purely electrostatic effect. The considerable effect of $\mathrm{Ba}^{2+}$ on the photodimerization efficiency of $\mathbf{3}$ can be rationalized by the formation of supramolecular assemblies in which two (or more) molecules of $\mathbf{3}$ are held together by simultaneously binding $\mathrm{Ba}^{2+}$ and 2 . Considering the 2:1 stoichiometry of the hydrogen bonding motif, it is plausible that the putative assembly is composed of $\mathbf{3}, \mathbf{2}$, and $\mathrm{Ba}^{2+}$ in a 2:1:1 ratio. Simultaneously binding both 2 and $\mathrm{Ba}^{2+}$ would effectively lock two molecules of $\mathbf{1}$ into a face-to-face geometry, which is known to be particularly favorable towards photodimerization. In contrast, if only one template is used, a greater number of geometrical isomers can be formed, and lowered reactivity is expected.

The systems described above find use in the development of photo-sensitive receptors for neutral molecules. In this case, light can be used to generate a wanted receptor in the presence of the selected target (substrate-induced receptor synthesis). The receptors are not only adapted to binding $\mathbf{2}$, the template used during irradiation, but are also capable of discriminating between such apparently similar nucleic acid derivatives as thymine and uracil, which is of interest for biological applications requiring discrimination between RNA and DNA [6]. The origin of this peculiar selectivity (uracil and thymine only differ by the presence of a single exocyclic methyl group) presumably lies in the rigidity imparted to the binding cleft by the cyclobutane structure.

In numerous cases, light has been used as a trigger to eject an ion bound to a crown ether appended with a photoactive chromophore by photomodulation of the electrostatic interactions via photoinduced charge transfer. In contrast, the photoejection of a bound neutral molecule is much more difficult to achieve as the latter requires a genuine reorganization of the binding cleft.

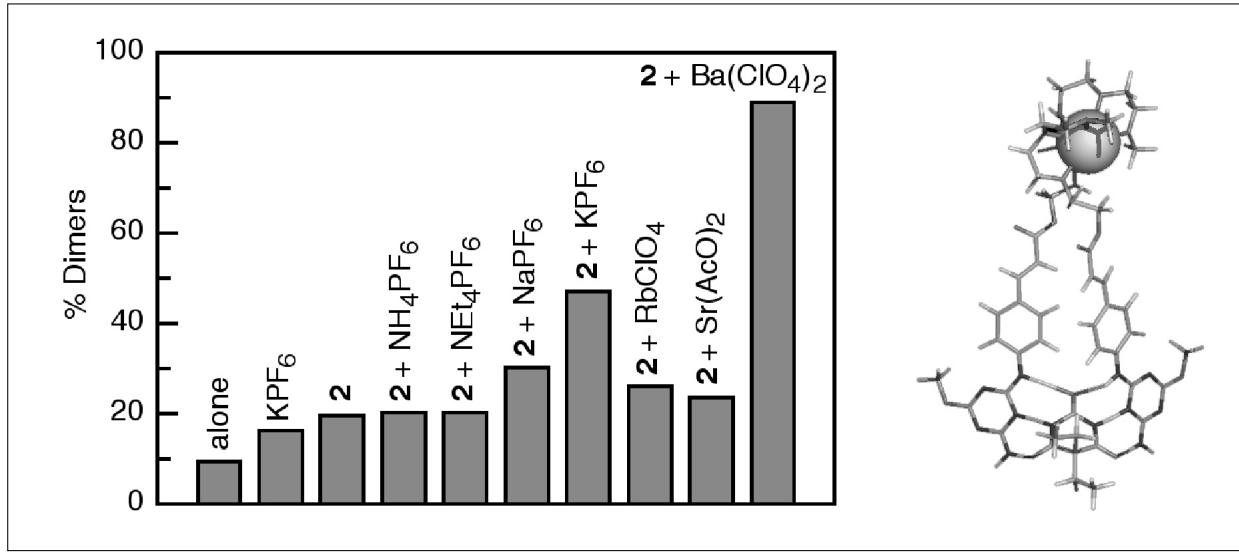

Fig. 2. Left: proportion of dimers (determined by HPLC) formed upon irradiation (400W Hanovia, Pyrex filter) of $3\left(0.01 \mathrm{M}\right.$ in $\left.\mathrm{CH}_{2} \mathrm{Cl}_{2}+5 \% \mathrm{CH}_{3} \mathrm{CN}\right)$ in the absence and presence of 2 and selected ions (0.5 equiv.). Right: Energy-minimized (PM3) structure of a 2:1:1 (3:2: $\left.\mathrm{Ba}^{2+}\right)$ complex in which the exocyclic $\mathrm{C}=\mathrm{C}$ bonds are proximal [7]

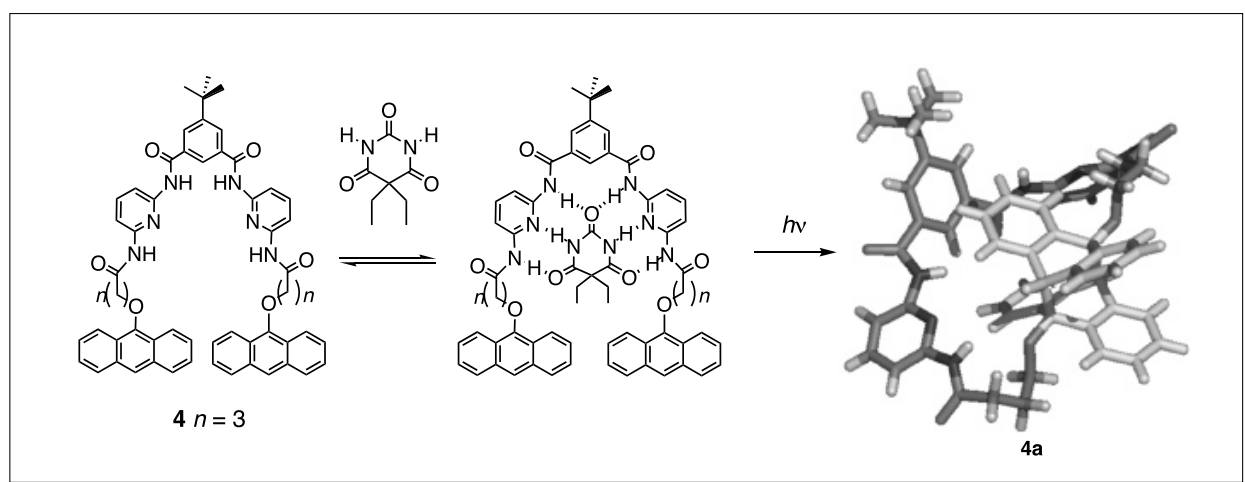

Fig. 3. By appending photodimerizable anthracene chromophores to a barbiturate receptor, it is possible to use light to eject bound barbital from the host. The crystal structure of the cyclized host 4a reveals that the binding cavity is blocked by the head-to-tail anthracene dimer [8].

This has been achieved in the case of compound 2 by substituting photodimerizable anthracene moieties on the side arms of the barbiturate receptor developed by Hamilton (Fig. 3). The spectacular modulation of $K_{\text {ass }}$ observed (from ca. $4 \times 10^{4}$ to $40 \mathrm{M}^{-1}$ for the open and cyclized form, respectively) is sufficient to provoke the ejection of the bound barbiturate upon illumination. The large increase in the substrate excluded molecular volume of the receptor is due to the formation of the head-to-tail anthracene photodimer, as evidenced by X-ray crystallographic analysis of the isolated photoproduct [8].

\section{Self-assembled Photoactive Devices}

Supramolecular self-assembly has played a major role in the move from isolated molecules to complex, interactive ensembles. A major feature of the self-assembly process is that individual components will spontaneously combine in a predetermined fashion due to the presence of complementary molecular recognition sites, and this route is often found to be much more efficient than conventional covalent chemistry for building large nanometersized assemblies. To use this strategy to prepare fullerene-containing architectures based on the melamine-barbituric acid system, we have designed a $\mathrm{C}_{60}$ appended with a barbituric acid molecular recognition site (compound 5, Fig. 4) [9].

As a test of the predisposition of 5 to form hydrogen-bonded architectures analogous to those described above, its intermolecular photodimerization as a possible first example of the intermolecular photodimerization of a fullerene derivative in solution. Irradiation of dilute $\left(5 \times 10^{-4} \mathrm{M}\right)$ solutions of 5 and a 1:1 mixture of 5 and a complementary melamine derivative in degassed $o$-dichlorobenzene $(o$-DCB) resulted in the gradual disappearance of $\mathbf{5}$ as judged by HPLC analysis. The irradiated solutions were then analyzed by MALDI-TOF MS using elemental sulfur as the matrix, where it can be seen that in the case of samples containing the melamine template, a clear signal for the $(\mathbf{5})_{2}$ dimer is observed. In the absence of template, no signals corresponding to the formation of either $\mathrm{C}_{60}$ dimer or 
$(\mathbf{5})_{2}$ could be detected by MALDI-TOF MS under identical irradiation conditions [9].

The tremendous amount of work aimed at harnessing the unique electronic properties of fullerenes in the design of new photovoltaic and sensor devices has been ongoing since their original discovery by Smalley and Kroto in 1985. In such devices, the fullerene acts as the electron acceptor in combination with an electron donor, such as a thiophene- or phenylenevinylene-based oligomer or polymer. Crucial requirements are both fast electron transfer from donor to acceptor, as well as efficient transport of electrons and holes amongst the donors and acceptors. For these applications the organization of the electron donating and electron accepting moieties is a prerequisite. The organization, which can be attained by making use of self-assembly, can lead to materials with very promising properties from an application point of view.

In the example shown in Fig. 5, a Hamilton-based receptor for barbituric acid is decorated with electron-rich oligothiophene-vinylene fragments. Upon binding $\mathbf{5}$, through-space interactions are such that an intense ground-state CT band is observed. The latter is indicative of strong electronic coupling. Excitation induces ultra-fast intra-assembly electron transfer $\left(k_{\mathrm{ET}}=5.5 \times\right.$ $\left.10^{12} \mathrm{~s}^{-1}\right)$, as determined by fs-laser flash photolysis studies [10].

Photovoltaic devices constructed using 5 and a triaminopyrimidine-appended oligothiophene are particularly efficient [11]. The molecular recognition groups greatly enhance compatibility between the otherwise poorly miscible fullerene and thiophene derivatives, thus enhancing the efficiency and the stability of the devices. Compared to control devices in which no hydrogen-bonding units are present, fivefold or greater APCE efficiency is obtained [11a]. This form of molecular recognition assisted self-assembly can be extended to even larger architectures, such as nanoparticles. Thus, semi-conducting $\mathrm{Au}$ and $\mathrm{CdS}$ nanoparticles decorated with thiol-appended barbiturate and melamine derivatives can self-assemble into ordered layers onto a gold electrode, which leads to enhanced photocurrent being observed [12].

\section{Conclusion}

In conclusion, the sensitivity of many photoinduced processes towards the molecular orientation of the reactants can be put to good use by designing tailored architectures which favor certain reaction pathways or exalt specific properties. This form of supramolecular control of excited state reactivity will certainly continue to draw attention due to the accrued interest in the design and fabrication of nanoscale devices

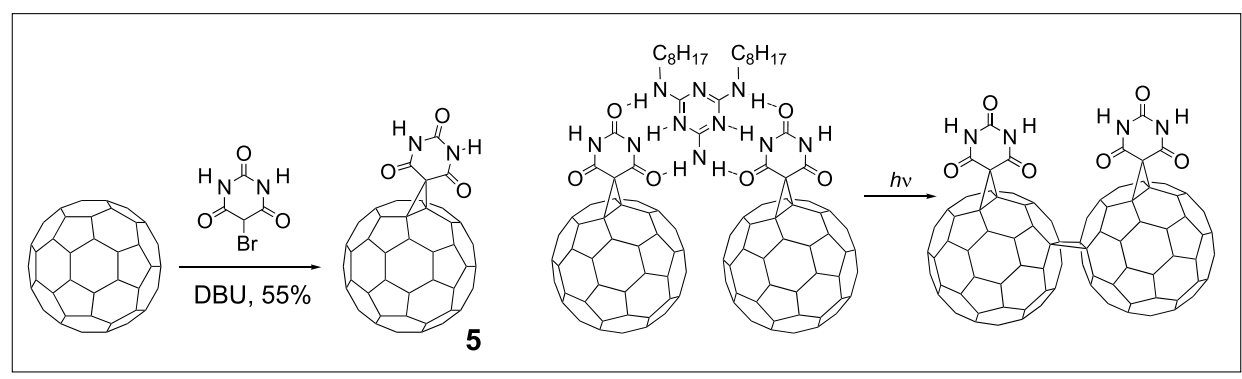

Fig. 4. A one step synthesis allows the efficient preparation of a fullerene derivative bearing a nonself-complementary hydrogen-bonding motif. The presence of a complementary tiaminopyrimidine template renders possible the intermolecular photodimerization of dilute solutions of 5 ( $0.5 \mathrm{mM}$ in o-dichlorobenzene) [9].

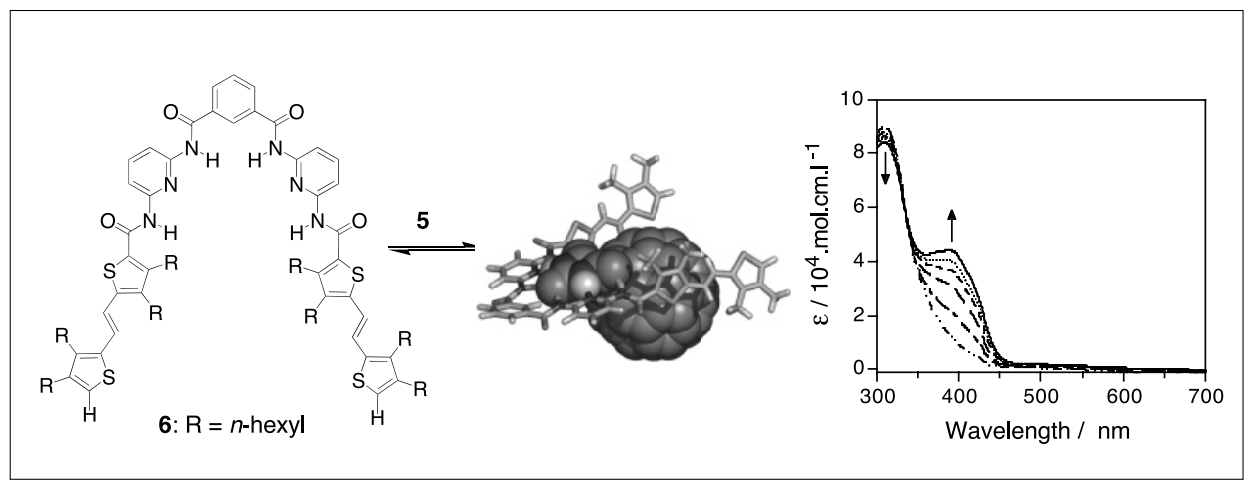

Fig. 5. Association of $\mathbf{5}$ within the cavity of $\mathbf{6}$ gives rise to strong through-space electronic coupling between the electroactive subunits, as evidenced by the observed molar extinction coefficient of 1:1 mixtures of $\mathbf{5}$ and $\mathbf{6}$ in $\mathbf{0}$-dichlorobenzene. The arrows indicate changes upon dilution of the solution [10].

operating at the molecular level, and the social and economic benefits associated with such high levels of miniaturization. The development of future applications notwithstanding, we and many others have found the study of fundamental processes in this area to be particularly stimulating and intellectually very rewarding.

Received: February 10, 2006

[1] a) E. Hertel, K. Schneider, Z. Elektrochem. 1931, 37, 536; b) M.D. Cohen, G.M.J. Schmidt, J. Chem. Soc. 1964, 2014, and references therein.

[2] D.M. Bassani, in 'CRC Handbook of Organic Photochemistry and Photobiology', 2nd ed., Eds. W.M. Horsepool, F. Lenci, CRC Press, Boca Raton, 2003, Ch. 20.

[3] For a recent review of the topic, see C.-H. Huang, D.M. Bassani, Eur. J. Org. Chem. 2005, 4041.

[4] a) D.M. Bassani, V. Darcos, S. Mahony, J.-P. Desvergne, J. Am. Chem. Soc. 2000, 122, 8795; b) V. Darcos, K. Griffith, X. Sallenave, J.-P. Desvergne, C. GuyardDuhayon, B. Hasenknopf, D.M. Bassani, Photochem. Photobiol. Sci. 2003, 11, 1152.

[5] a) D. Faure, J. Gravier, T. Labrot, B. Desbat, R. Oda, D.M. Bassani, Chem. Commun. 2005, 1167; b) L. Goracci, R. Germani, G. Savelli, D.M. Bassani, Chem. BioChem. 2005, 6, 197.
[6] D.M. Bassani, X. Sallenave, V. Darcos, J.-P. Desvergne, Chem. Commun. 2001, 1446.

[7] Y. Vida Pol, R. Suau, E. Perez-Inestrosa, D.M. Bassani, Chem. Commun. 2004, 1270.

[8] Y. Molard, D.M. Bassani, J.-P. Desvergne, P.N. Horton, M.B. Hursthouse, J.H.R. Tucker, Angew. Chemie, Int. Ed. 2005, 44, 2.

[9] N.D. McClenaghan, C. Absalon, C. D.M. Bassani, J. Am. Chem. Soc. 2003, 125, 13004.

[10] N.D. McClenaghan, Z. Grote, D. Darriet, M. Zimine, R.M. Williams, L. De Cola, D.M. Bassani, Org. Lett. 2005, 7, 807.

[11] a) C.-H. Huang, N.D. McClenaghan, A. Kuhn, J.W. Hofstraat, D.M. Bassani, Org. Lett. 2005, 7, 3409; b) C.-H. Huang, N.D. McClenaghan, A. Kuhn, G. Bravic, D.M. Bassani, Tetrahedron 2006, in press (Special issue on fullerenes).

[12] R. Baron, C.-H. Huang, D.M. Bassani, A. Onopriyenko, M. Zayats, I. Willner, Angew. Chemie, Int. Ed. 2005, 44, 4010. 